\title{
International Spinal Cord Injury Lower Urinary Tract Function Basic Data Set (version 2.0)
}

\author{
Fin Biering-Sørensen ${ }^{1} \cdot$ Michael Kennelly ${ }^{2}$ Thomas M. Kessler ${ }^{3} \cdot$ Todd Linsenmeyer $^{4,5} \cdot$ Jürgen Pannek $^{6}$. \\ Lawrence Vogel $^{7,8} \cdot$ Jean-Jacques Wyndaele ${ }^{9}$
}

Received: 16 April 2018 / Revised: 3 June 2018 / Accepted: 4 June 2018

(C) International Spinal Cord Society 2018

\begin{abstract}
Study design Revision, review, and presentation of the International Spinal Cord Injury (SCI) Lower Urinary Tract (LUT) Function Basic Data Set (version 2.0).

Objectives Describe the revision and review and present the data set.

Setting International.

Methods The first version of the data set was revised according to new knowledge and suggestions. The review included International SCI Data Sets Committee, American Spinal Injury Association (ASIA) board, International Spinal Cord Society (ISCoS) executive and scientific committees, major organizations, and interested individuals. The data set was also on ASIA and ISCoS websites. All replies were answered and appropriate adjustments made. Finally, the data set was endorsed by ASIA board, and ISCoS executive and scientific committees.

Results Among revisions are adoptions of new terminology by the International Continence Society. For most variables, advice for collection of information from pediatric patients stated. For the variable 'Bladder emptying', is in the data collection form to the response category 'Normal voiding' expanded. 'Sacral Anterior Root Stimulator' is deleted as response category. For the variable 'Any involuntary urine leakage (incontinence) within the last 4 weeks' 'last 4 weeks' has replaced 'last 3 months'. The response categories have been adjusted to: 'Daily', 'Once or more per week (but not daily)', 'Less than once per week', 'Never', 'Not applicable' and 'Unknown'. For the variable 'Any drugs for the urinary tract within the last four weeks' 'last four weeks' has replaced 'last year'.

Conclusions The International SCI LUT Function Basic Data Set (version 2.0) with its complete syllabus is available from http://www.iscos.org.uk/international-sci-data-sets.
\end{abstract}

Electronic supplementary material The online version of this article (https://doi.org/10.1038/s41394-018-0090-7) contains supplementary material, which is available to authorized users.

Fin Biering-Sørensen

fin.biering-soerensen@ regionh.dk

1 Clinic for Spinal Cord Injuries, Rigshospitalet, University of Copenhagen, Copenhagen, Denmark

2 Carolinas Rehabilitation, Carolinas Healthcare System, Charlotte, NC, USA

3 Neuro-Urology, Spinal Cord Injury Center \& Research, University of Zürich, Balgrist University Hospital, Zürich, Switzerland

\section{Introduction}

The first version of the International Spinal Cord Injury (SCI) Lower Urinary Tract (LUT) Function Basic Data Set was published in 2008 [1]. The purpose of this data set is to standardise the collection and reporting of a minimum amount of information on the LUT in daily clinical practice

4 Urology Department, Kessler Institute for Rehabilitation, W. Orange, NJ, USA

5 Departments Surgery (Urology) and PM\&R, Rutgers New Jersey Medical School, Newark, NJ, USA

6 Neuro-Urology, Swiss Paraplegic Centre, Nottwil, Switzerland

7 Shriners Hospitals for Children, Chicago, IL, USA

8 Rush University, Chicago, IL, USA

9 University Antwerp, Antwerp, Belgium 
in accordance with the purpose and vision of the International SCI Data Sets [2]. This will make it possible to evaluate and compare results from various published studies.

All International SCI Data Sets undergo periodic review to ensure continued relevance, acceptance and usage by the SCI clinical/research community. In addition, the International SCI Data Sets were reviewed for their relevance for pediatric SCI. The aim of this presentation is to describe the revision and review process, and present the final updated version of the International SCI LUT Basic Data Set (Version 2.0).

\section{Methods}

The first version of the International SCI LUT Basic Data Set was revised according to new knowledge and suggestions received from the SCI community. The review included consultation with the International SCI Data Sets Committee, the American Spinal Injury Association (ASIA) board, the International Spinal Cord Society (ISCoS) executive and scientific committees, major related national and international organizations, and interested individuals who asked to be on a reviewer mailing list. In addition, the data set were posted on ASIA and ISCoS websites for a month inviting everyone to submit comments.

All suggestions were evaluated and responded to by the Working Group (the authors) and adjustments made if found appropriate. When reviewing proposed revisions, the potential benefits of the proposal were weighed against the loss of continuity resulting from any revision. These changes, apart from minor corrections, are summarised below in the revised comments to the variables and in the data collection form in the Appendix.

After the revision and review, the final International SCI LUT Basic Data Set (Version 2.0) was endorsed by the ASIA board and the ISCoS executive and scientific committees and made freely available with the complete syllabus at http://www.iscos.org.uk/international-sci-data-sets.

\section{Results}

The International Continence Society (ICS) has made an update on the terminology for adult neurogenic lower urinary tract dysfunction [3]. As a consequence, the wording of the comments are adjusted as given below for the following variables: 'Awareness of the need to empty the bladder', 'Bladder emptying', 'Any involuntary urine leakage (urinary incontinence) within the last four weeks', and 'Any change in lower urinary tract symptoms within the last year.'
For the pediatric age groups, for most variables it is stated: "This variable is appropriate for all pediatric age groups $0-5,6-12,13-14$, and 15-17. If collecting information from pediatric patients, self-report will be primarily by parents for age groups $0-5$; and primarily from the child for age groups 6-12 (parent report, secondary), 13-14 (parent report, supplemental), and 15 and older (parent report, optional)." For the variable "Awareness of the need to empty the bladder', the wording is a little different and given specifically.

Below the variables with the adjustments are given.

\section{Urinary tract impairment unrelated to spinal cord lesion}

This variable documents any type of urinary tract impairment unrelated to the spinal cord lesion on the date of data collection.

To be able to evaluate the LUT function in an individual with spinal cord lesion, it is necessary to know if there are any other urinary tract impairments unrelated to the spinal cord lesion.

If there have been any urinary tract impairments unrelated to the spinal cord lesion, this/these is/are recommended to be written in a text-field, from which it will be possible to retrieve more detailed data when necessary. Because many possible urinary tract impairments exist, it is not practical to give an exact list of impairments.

If this information has been documented once and no additional urinary tract impairments unrelated to the spinal cord lesion have been identified it is only necessary to complete this item once, to avoid redundant data.

\section{Awareness of the need to empty the bladder}

This variable documents awareness of the need to empty the bladder on the date of data collection.

Awareness of the need to empty the bladder indicates any kind of bladder sensation reported during history taking as defined by ICS [3, 4], i.e., normal bladder sensation (awareness of bladder filling and increasing sensation up to a strong desire to void), reduced bladder sensation (definite desire to void occurs later to that previously experienced despite an awareness that the bladder is filling), increased bladder sensation (the desire to void during bladder filling occurs earlier or is more persistent to that previous experienced. This differs from urgency by the fact that micturition can be postponed despite the desire to void.), abnormal bladder sensation (e.g., "tingling", "burning", or "electric shock"), or non-specific bladder awareness (perceive bladder filling as abdominal fullness, vegetative symptoms like sweating (as part of autonomic dysreflexia), urethral sensations or spasticity) [3, 4]. 
No awareness of the need to empty the bladder should be noted as "no". Absent bladder sensation, according to the definition of bladder sensation by the ICS, i.e., the individual reports no sensation of bladder filling or desire to void [3, 4].

"Not applicable" is to be used when the individual has, for example, an unclamped indwelling catheter or noncontinent urinary diversion.

This variable is appropriate for all pediatric age groups older than 3 years. If collecting information from pediatric patients, parents are the primary source of report for 3-5; and self-report primarily from the child for age groups 6-12 (parent report, secondary), 13-14 (parent report, supplemental), and 15 and older (parent report, optional)." However, for very young children (birth to 3 years) this variable is "Not applicable".

\section{Bladder emptying}

This variable documents the method(s) used to empty the bladder on the date of data collection. For each method of bladder, emptying it is possible to indicate whether this is a main or a supplementary method. Two main and one or more supplementary methods may be indicated - adopted from Levi and Ertzgaard [5].

\section{Normal voiding}

Voluntary initiation of micturition without reflex stimulation or compression of the bladder. This does not presume entirely normal function [5]. This is included in the data collection form of version 2.0 to ensure that the data collector is aware of this fact.

\section{Bladder reflex triggering}

Bladder reflex triggering comprises various manoeuvres performed by the individual with spinal cord lesion or an attendant in order to elicit reflex detrusor contraction by exteroceptive stimuli. The most commonly used manoeuvres are suprapubic tapping, thigh scratching and anal/ rectal manipulation [4].

Voluntary bladder reflex triggering indicates that the bladder reflex is triggered by the individual with spinal cord lesion him/herself or by the attendant.

Involuntary bladder reflex triggering implies that there is no voluntary triggering of the voiding, but the individual with spinal cord lesion allows the urine to pass by itself when the reflex detrusor contraction occurs.

\section{Bladder expression}

Bladder expression comprises various manoeuvres aimed at increasing intravesical pressure in order to facilitate bladder emptying. The most commonly used manoeuvres are abdominal straining, Valsalva's manoeuvre and external manual suprapubic pressure (Credé manoeuvre) [3, 4].

Straining includes abdominal straining, Valsalva's manoeuvre.

External compression includes Credé manoeuvre.

Catheterisation is a technique for bladder emptying employing a catheter to drain the bladder or urinary reservoir [4].

\section{Intermittent catheterisation}

Intermittent catheterisation is by the ICS defined as drainage of the bladder or a urinary reservoir with subsequent removal of the catheter mostly at regular intervals [3].

In the International SCI LUT Function Basic Data Set (version 2.0), the following types of intermittent catheterisation can be reported:

Intermittent self-catheterisation is performed by the individual himself/herself.

Intermittent catheterisation can also be performed by an attendant (e.g. family member or personal aid).

This means that there will not be a distinction between the techniques used and defined by the ICS, i.e. clean, aseptic, sterile or no-touch technique intermittent catheterisation [3].

\section{Indwelling catheterisation}

An indwelling catheter remains in the bladder, urinary reservoir or urinary conduit for a period longer than one emptying [3, 4].

In the International SCI LUT Function Basic Data Set (version 2.0) the following types of indwelling catheterisation can be reported:

Transurethral indwelling catheterisation indicates that the urine is drained trough a catheter placed in the urethra.

Suprapubic indwelling catheterisation indicates that the urine is drained through a catheter via the abdominal wall.

\section{Non-continent urinary diversion/ostomy}

This includes ureteroileocutaneostomy (ileoureterostomy, ileal loop, Bricker conduit, ileal conduit), ileovesicostomy, and vesicostomy.

If any Other method is used for bladder emptying, it is recommended to be written in a text-field, from which it will be possible to retrieve more detailed data when necessary. Because other methods of bladder emptying, including sacral anterior root stimulators, which in the first version had a separate response category, are generally rare, and therefore it is not practical to give an inclusive list of other bladder emptying methods. 
Average number of voluntary bladder emptyings per $24 \mathrm{~h}$ during the last week.

This variable documents the average number of voluntary bladder emptyings per $24 \mathrm{~h}$ during the last week. 'Per day' in the first version has been replaced by'per $24 \mathrm{~h}$ ' in version 2.0 to specify it to mean day and night.

The average number of voluntary bladder emptying per $24 \mathrm{~h}$ during the last week is given separately. This number refers to the number of voluntary bladder emptyings irrespective of the method. Any of the following methods may be used separately or in combination: normal voiding, voluntary bladder reflex triggering, bladder expression, intermittent catheterisation, or others. If a combination of methods is used during the same bladder emptying it should be counted as only one bladder emptying. The number is given as an average for the last week, as the individual is not expected to remember this for a longer period of time. The number is given as the nearest integer number.

"Not applicable" was introduced in version 2.0 for individuals using an indwelling catheter. "Unknown" was also introduced in version 2.0 for someone like a child or an adult who wear diapers/nappies/incontinence briefs and may not be aware of the number of bladder emptyings.

\section{Any involuntary urine leakage (urinary incontinence) within the last four weeks}

This variable documents the average involuntary urine leakage (urinary incontinence) within the last 4 weeks prior to the date of data collection. 'Last 3 months' in the first version of the data set has been replaced by "last four weeks" in version 2.0 to align this data set with the time frame consistent across the various International SCI Data Sets. In addition, the response categories have been adjusted to: "Daily", "Once or more per week (but not daily)", "Less than once per week", "Never".

Urinary incontinence is defined by the ICS [3,4] as the "complaint" of involuntary loss of urine. In each specific circumstance the urinary incontinence should be further described by specifying relevant factors such as type (stress, urgency, mixed urinary incontinence or enuresis), frequency, severity, precipitating factors, social impact, effect on hygiene and quality of life, etc. [3, 4]. In the International SCI LUT Function Basic Data Set (version 2.0), only a simple indication of severity and collection of urine is given.

Bladder reflex triggering, including into a collection system, e.g., condom catheter may be voluntary and thus not considered as incontinence. However, if the condom or ostomy bag falls off and the individual "complains" of incontinence then it should be recorded as "Yes".
Involuntary urine leakage (urinary incontinence) within the last 4 weeks implies leakage of urine outside the urinary tract or a closed urinary collection system.

"Not applicable" may be used when the individual has, for example, a non-continent urinary diversion.

\section{Collecting appliances for urinary incontinence}

This variable documents use of any collecting appliances for urinary incontinence on the date of data collection.

Collecting appliances are any externally applied aids to avoid urinary leakage, or devices for collection of urine. Regular use of one or more collecting appliances is to be recorded. Individuals that use such appliances less than once a month, "for the sake of safety", and who have no more than exceptional episodes of leakage during a year should be coded as "No" (adapted from [5]).

For other possible collecting appliances, it is recommended to write them in a text-field, from which it will be possible to retrieve more detailed data when necessary.

\section{Any drugs with possible influence on the urinary tract within the last four weeks}

This variable documents use of any drugs, systemic or intravesical, with possible influence on the urinary tract within the last four weeks on the date of data collection. 'Last year' in the first version has been replaced within the 'last four weeks' in version 2.0, as it is hard reliably to recall a time period of one year and additionally to align this data set with the time frame consistent across the various International SCI Data Sets.

Bladder relaxant drugs, i.e., drugs causing relaxation of the detrusor, include antimuscarinics, anticholinergics, tricyclic antidepressant, etc. These drugs may also be delivered intravesically. This does not include treatment with injections into the detrusor.

Sphincter and bladder neck relaxant drugs include alpha adrenergic blockers, etc. This does not include treatment with injections into the sphincter.

Antibiotics and antiseptics used for treatment or prophylaxis of urinary tract infections are coded separately. For prevention of urinary tract infections, drugs such as methenamine are included. For other possible drugs, it is recommended to write them in a text-field, from which it will be possible to retrieve more detailed data when necessary.

\section{Surgical procedures on the urinary tract}

This variable documents any surgical procedures on the urinary tract up to the date of data collection. If more than 
one procedure of the same kind has been performed only the last one is documented with date of performance.

Bladder stone or upper urinary tract stone removal includes any type of removal, including via endoscopy, extracorporal shock wave lithotripsy (ESWL), or open lithotomy.

The ileoureterostomy (ileal conduit) is the same as ileal loop or ureteroileocutaneostomy (Bricker conduit). In version 2.0'(ileal conduit)' has been included to be consistent with the most common wording used in the urological literature [6-8].

The continent catheterizable valves, include the Monti and the Mitrofanoff procedures [8].

For other possible surgical procedures, including sacral anterior stimulator [6-8], which is now rarely implanted and in the first version had a separate response category, it is recommended to write them in a text-field, from which it will be possible to retrieve more detailed data when necessary. If there is more than one "other" surgical procedure, this field may be duplicated or triplicated, with the latest date given when the particular procedure has been performed.

If the information has been documented once and no additional surgical procedures on the urinary tract have been performed, it is not necessary fill in this item again to avoid redundant data.

\section{Any change in lower urinary tract symptoms within the last year}

This variable documents any change in LUT symptoms (LUTS) within the last year from the date of data collection.

According to the ICS, LUTS are the subjective indicator of a disease or change in conditions as perceived by the individual with spinal cord lesion, attendant or partner, and may lead him/her to seek help from health care professionals [3]. Symptoms may be described during the data collection interview with the individual with spinal cord lesion. The information is usually qualitative, and may include change in frequency, urgency, nocturia, incontinence, hesitancy, slow stream, etc. LUTS can in individuals with spinal cord lesion indicate urinary tract infection even when bacteriuria has no associated signs or symptoms. Chills and fever are often considered to be signs of acute pyelonephritis; however, these signs do not confirm an infection in the upper urinary tract [9]. Nonetheless, chills and fever may be the only symptoms and signs experienced by people with a spinal cord lesion and pyelonephritis, bacteremia, upper tract obstruction by calculi, renal abscess, and perinephric abscess. Other suspicious signs and symptoms may include increased sweating, abdominal discomfort, costovertebral angle pain or tenderness, and increased muscle spasticity [9]. Cloudy and malodorous urine and changes in urine $\mathrm{pH}$ may be signs of urinary tract infection, but can also occur with colonisation, changes of bacterial organisms, and various food intake. Increased spontaneous voiding or larger residual urines including acute urinary retention may be seen with acute infection [9].

"Not applicable" is to be used when data reporting is performed within the first year after the spinal cord lesion.

\section{Discussion}

Collection of data on LUT function is strongly encouraged when individuals with spinal cord lesions consult doctors with knowledge regarding spinal cord lesions.

The reliability of the first version of the data set was tested and found satisfactory [10]. Further it is to be appreciated that many of the data information included in the International SCI LUT Basic Data Set are according to the ICS recommendations [3], which are to be as is to maintain consistency with international standards already in place.

The data in this International SCI LUT Function Basic Data Set (version 2.0) generally will be used with data in the International SCI Core Data Set (Version 2.0) [11], which includes information on date of birth and injury, gender, the cause of spinal cord lesion, and neurologic status. Other related International SCI Data Sets including urological information are the International SCI Urinary Tract Infection Basic Data Set [12], the International SCI Urodynamic Basic Data Set [13] (being updated), and the International SCI Urinary Tract Imaging Basic Data Set [14].

A spinal cord lesion may be traumatic or non-traumatic in aetiology. All lesions to the spinal cord, conus medullaris, and cauda equina are included in the present context.

It is extremely important that the data be collected in a uniform manner. For this reason, each variable and each response category within each variable has been defined to promote the collection and reporting of comparable minimal data.

Use of a standard format is essential for combining the data from multiple investigators and locations. Various formats and coding schemes may be equally effective and could be used in individual studies or by agreement of the collaborating investigators.

The International SCI LUT Function Basic Data Set (version 2.0) with its complete syllabus is available from http://www.iscos.org.uk/international-sci-data-sets.

The International SCI LUT function Basic Data Set is already incorporated in the data registries/electronic medical records in Australia, the Netherlands [15, 16], Canada, Norway, Denmark, and probably at other locations as well, as translations exist to several other languages. In addition, the electronic medical record Epic (Verona, Wisconsin, 
USA) will be implementing it in their Foundation System in 2018, and thus Epic customers worldwide, including several SCI centers in the United States of America, Canada, United Kingdom, and Denmark will be able to use this [17]. Further, is it increasingly being implemented in clinical studies [15, 18, 19], and has been included in the National Institute of Health, National Institute of Neurological Disorders and Stroke, the Common Data Element project (https://www.commondataelements.ninds.nih.gov/SCI. aspx\#tab = Data_Standards) suggesting it to be used whenever appropriate in specific SCI studies.

Acknowledgements We are thankful for comments and suggestions for International SCI LUT Function Data Set (version 2.0) received from Meier Sonja, Susan Charlifue, Eva Widerström-Noga, Lyn Jakeman, Marcel Post, Vanessa Noonan, and Thomas Bryce.

Funding No support or funding was received for the development of the International SCI LUT Function Basic Data Set version 2.0.

\section{Compliance with ethical standards}

Conflict of interest The authors declare that they have no conflict of interest.

\section{References}

1. Biering-Sørensen F, Craggs M, Kennelly M, Schick E, Wyndaele JJ. International Lower Urinary Tract Function Basic Spinal Cord Injury Data Set. Spinal Cord. 2008;46:325-30.

2. Biering-Sørensen F, Charlifue S, DeVivo M, Noonan V, Post M, Stripling T, Wing P. International spinal cord injury data sets. Spinal Cord. 2006;44:530-4.

3. Gajewski JB, Schurch B, Hamid R, Averbeck M, Sakakibara R, Agrò EF, Dickinson T, Payne CK, Drake MJ, Haylen BT. An International Continence Society (ICS) report on the terminology for adult neurogenic lower urinary tract dysfunction (ANLUTD). Neurourol Urodyn. 2018;37:1152-61. https://doi.org/10.1002/na u. 23397

4. Abrams P, Cardozo L, Fall M, Griffiths D, Rosier P, Ulmsten U, van Kerrebroeck P, Victor A, Wein A. The Standardisation of terminology of lower urinary tract function: report from the standardisation sub-committee of the international continence society. Neurourol Urodyn. 2002;21:167-78.

5. Levi R,Ertzgaard P, The Swedish Spinal Cord Injury Council 1998. Quality indicators in spinal cord injury care: A Swedish collaboration project. Scand J Rehabil Med. 1998;38:1-80.

6. Panicker JN, Fowler CJ, Kessler TM. Lower urinary tract dysfunction in the neurological patient: clinical assessment and management. Lancet Neurol. 2015;14:720-32. https://doi.org/10. 1016/S1474-4422(15)00070-8

7. Groen J, Pannek J, Castro Diaz D, Del Popolo G, Gross T, Hamid R, Karsenty G, Kessler TM, Schneider M. 't Hoen L, Blok B. Summary of European Association of Urology (EAU) Guidelines on Neuro-Urology. Eur Urol. 2016;69:324-33. https://doi.org/10. 1016/j.eururo.2015.07.071
8. Wyndaele JJ, Birch B, Borau A, Burks F, Castro-Diaz D, ChartierKastler E, Drake M, Ishizuka O, Minigawa T, Opisso E, Peters K, Padilla-Fernández B, Reus C, Sekido N. Surgical management of the neurogenic bladder after spinal cord injury. World J Urol. 2018. https://doi.org/10.1007/s00345-018-2294-7.

9. Stover SL, Lloyd K, Waites KB, et al. Urinary tract infection in spinal cord inury. Arch Phys Med Rehabil. 1989;70:47-54.

10. Nachtegaal J, van Langeveld SA, Slootman H, Post MWM. Dutch-flemish spinal cord society. implementation of a standardized dataset for collecting information on patients with spinal cord injury. Top Spinal Cord Inj Rehabil. 2018;24:133-40. https:// doi.org/10.1310/sci2402-133.

11. Biering-Sørensen F, DeVivo MJ, Charlifue S, Chen Y, New PW, Noonan V, Post MWM, Vogel L. International Spinal Cord Injury Core Data Set (version 2.0) - including standardization of reporting. Spinal Cord. 2017;55:759-64. https://doi.org/10.1038/ sc. 2017.59

12. Goetz LL, Cardenas DD, Kennelly M, Bonne Lee BS, Linsenmeyer T, Moser C, Pannek J, Wyndaele JJ, Biering-Sorensen F. International spinal cord injury urinary tract infection basic data set. Spinal Cord. 2013;51:700-4. https://doi.org/10.1038/sc.2013. 72

13. Biering-Sørensen F, Craggs M, Kennelly M, Schick E, Wyndaele JJ. International urodynamic basic spinal cord injury data set. Spinal Cord. 2008;46:513-6.

14. Biering-Sørensen F, Craggs M, Kennelly M, Schick E, Wyndaele J-J. International urinary tract imaging basic spinal cord injury data set. Spinal Cord. 2009;47:379-83.

15. Adriaansen JJ, van Asbeck FW, Tepper M, Faber WX, VisserMeily JM, de Kort LM, Post MW. Bladder-emptying methods, neurogenic lower urinary tract dysfunction and impact on quality of life in people with long-term spinal cord injury. J Spinal Cord Med. 2017;40:43-53. https://doi.org/10.1179/2045772315Y. 0000000056

16. Post MWM, Nachtegaal J, van Langeveld SA, van de Graaf M, Faber WX, Roels EH,van Bennekom CAM. Progress of the Dutch Spinal Cord Injury Database: completeness of database and profile of patients admitted for inpatient rehabilitation in 2015. Top Spinal Cord Inj Rehabil. 2018;24:141-50. https://doi.org/10.1310/ sci2402-141

17. Biering-Sørensen F, Cohen S, Rodriguez GM, Tausk K, Martin J. Electronic Medical Record: Data Collection and Reporting for Spinal Cord Injury (SCI). Including International SCI Data Sets and Standards for Neurological Classification of SCI. Spinal Cord Ser Cases. 2018

18. Hubscher $\mathrm{CH}$, Herrity AN, Williams CS, Montgomery LR, Willhite AM, Angeli CA, Harkema SJ. Improvements in bladder, bowel and sexual outcomes following task-specific locomotor training in human spinal cord injury. PLoS ONE. 2018;13: e0190998 https://doi.org/10.1371/journal.pone.0190998. eCollection 2018

19. Bach Baunsgaard C, Vig Nissen U, Katrin Brust A, Frotzler A, Ribeill C, Kalke YB, León N, Gómez B, Samuelsson K, Antepohl W, Holmström U, Marklund N, Glott T, Opheim A, Benito J, Murillo N, Nachtegaal J, Faber W, Biering-Sørensen F. Exoskeleton gait training after spinal cord injury: Effect on pain, spasticity, range of motion, bowel- and lower urinary tract function, Spinal Cord Independence Measure III, and quality of life. Annu Conf Int Spinal Cord Soc. 2017. 